\title{
Noise-Induced Scenario for Inverted Phase Diagrams
}

\author{
M. Ibañes, ${ }^{1}$ J. García-Ojalvo, ${ }^{2}$ R. Toral, ${ }^{3}$ and J. M. Sancho ${ }^{1}$ \\ ${ }^{1}$ Department d'Estructura i Constituents de la Matèria, Universitat de Barcelona, Diagonal 647, E-08028 Barcelona, Spain \\ ${ }^{2}$ Departament de Física i Enginyeria Nuclear, Universitat Politècnica de Catalunya, Colom 11, E-08222 Terrassa, Spain \\ ${ }^{3}$ Instituto Mediterráneo de Estudios Avanzados (CSIC-UIB), E-07071 Palma de Mallorca, Spain
}

(Received 31 October 2000; published 22 June 2001)

\begin{abstract}
We introduce a class of exactly solvable models exhibiting an ordering noise-induced phase transition in which order arises as a result of a balance between the relaxing deterministic dynamics and the randomizing character of the fluctuations. A finite-size scaling analysis of the phase transition reveals that it belongs to the universality class of the equilibrium Ising model. All these results are analyzed in the light of the nonequilibrium probability distribution of the system, which can be obtained analytically. Our results could constitute a possible scenario of inverted phase diagrams in the so-called lower critical solution temperature transitions.
\end{abstract}

DOI: $10.1103 /$ PhysRevLett.87.020601

A surprising discovery in the field of stochastic processes is that the interaction between noise and spatial degrees of freedom in extended nonlinear systems can lead, under appropriate conditions, to noise-induced order [1]. This seemingly counterintuitive phenomenon arises when a distributed medium develops some kind of spatiotemporal self-organization which requires noise to exist. The resulting ordered state can take many forms, such as a static spatial pattern [2], traveling excitable waves [3], propagating pulses [4], spatiotemporal chaotic states [5], or a coherent response to an external periodic forcing [6], among others. From a fundamental point of view, however, the most interesting example is that of noise-induced ordering phase transitions (NIOPTs) [7]. In this case, transitions between true extended phases (in a thermodynamic sense) are produced when the noise intensity is used as a control parameter. These nonequilibrium phase transitions can therefore be characterized using standard techniques of critical phenomena, such as dynamic renormalization group, mean-field approximations, as well as finite-size scaling analyses using, for instance, results obtained from numerical simulations of stochastic partial differential equations. Until now, all the arguments presented to account for the occurrence of NIOPTs have been dynamical ones. This is mainly because it has been impossible thus far to find a nonequilibrium model whose steady-state probability distribution and the associated effective potential are known. In particular, noise-induced phase transitions have been systematically explained in terms of a short-time instability of the local dynamics, which becomes "frozen" at larger times by the spatial coupling $[7,8]$.

In this Letter, we introduce a class of systems exhibiting NIOPTs for which the steady-state probability distribution can be obtained exactly, so that one can define the corresponding nonequilibrium free energy or potential. As a consequence, the NIOPT can be studied in the steady state, with no dynamical reference. It turns out that the noiseinduced phase transition that appears in these model systems is not a consequence of an instability of the disordered
PACS numbers: 05.40.-a, 05.10.Gg, 64.60.-i

homogeneous state. Rather, the situation is similar to what happens for noise-induced transitions in zero-dimensional systems, where the disordered state is linearly stable, but the effective nonequilibrium potential in the steady state is bimodal $[9,10]$. A phase transition is obtained by coupling adequately many of those zero-dimensional systems. For a strong enough spatial coupling, the neighboring variables tend to a common value and a macroscopic ordered phase appears as a result of ergodicity breaking. This situation contrasts vividly with NIOPTs reported thus far, where the need of a short-time dynamical instability prevents systems that undergo noise-induced transitions in $0 \mathrm{D}$ from exhibiting NIOPTs in the presence of spatial coupling [7].

The phase transition that we report in what follows shares some features with the so-called lower critical solution temperature (LCST) transition which appears in some polymer blends [11]. In these very special systems, one can go from a disordered phase (homogeneous mixture) to an ordered phase (separated phases) by increasing the temperature (inverted phase diagram). This phenomenology goes in the opposite direction of what is expected intuitively from statistical physics. Our findings present a possible scenario, in terms of a Langevin description, in which these transitions can take place. To reach our goal, we consider two important theoretical ingredients: the presence of a field-dependent kinetic coefficient and the existence of a fluctuation-dissipation relation accordingly.

Let us consider the following generic deterministic model for a real field $\phi(\vec{r}, t)$ :

$$
\frac{\partial \phi(\vec{r}, t)}{\partial t}=-\Gamma[\phi(\vec{r}, t)] \frac{\delta V}{\delta \phi(\vec{r}, t)},
$$

which corresponds to a relaxational flow [12] in a potential $V(\{\phi\})$ with a field-dependent kinetic coefficient $\Gamma(\phi)$. Field-dependent kinetic coefficients can appear in coarse-grained, master-equation derivations of macroscopic (deterministic or stochastic) field equations $[13,14]$. 
Their explicit form depends on the assumptions in their derivation, but the general trend is that the coefficient is large in the disordered phase and small in the ordered phase $[14,15]$. These coefficients have been introduced in the context of phase separation in binary mixtures, either to explore $a d$ hoc their influence, or by necessity to take into account the effect of a gravitational field [16]. In those cases, to reflect the conserved nature of the concentration field, the kinetic coefficients are also a function of the spatial derivatives of the field. Here, on the other hand, we consider for simplicity a nonconserved situation.

When stochastic terms are introduced in this description a fluctuation-dissipation relation is used [14]. Following this approach, we define the Langevin equation (in the Stratonovich interpretation) corresponding to the deterministic equation (1) as

$$
\begin{aligned}
\frac{\partial \phi(\vec{r}, t)}{\partial t}= & -\Gamma[\phi(\vec{r}, t)] \frac{\delta V}{\delta \phi(\vec{r}, t)} \\
& +\Gamma[\phi(\vec{r}, t)]^{1 / 2} \xi(\vec{r}, t) .
\end{aligned}
$$

The noise is taken to be Gaussian, with zero mean and correlation,

$$
\left\langle\xi(\vec{r}, t) \xi\left(\vec{r}^{\prime}, t^{\prime}\right)\right\rangle=2 \varepsilon \delta\left(\vec{r}-\vec{r}^{\prime}\right) \delta\left(t-t^{\prime}\right),
$$

where $\varepsilon$ is the noise intensity. Under these conditions, the stationary solution of the probability distribution for the field $P_{s t}(\{\phi\})$ is of the Boltzmann's type:

$$
P_{s t}(\{\phi\}) \sim e^{-V_{\text {eff }} / \varepsilon},
$$

in terms of an effective potential,

$$
V_{\mathrm{eff}}(\{\phi\}) \equiv V(\{\phi\})+\frac{\varepsilon_{0}}{2} \int d \vec{r} \ln \Gamma[\phi(\vec{r})],
$$

where $\varepsilon_{0}$ is a renormalized parameter, proportional to $\varepsilon$, which includes an ultraviolet cutoff [17]. The previous expression defines the exact nonequilibrium free energy of model (2). We will now show that this class of systems can exhibit a noise-induced phase transition.

Thus far, the discussion has been made in very general terms. In order to proceed further, we assume that the deterministic free-energy potential has the usual Landau form,

$$
V(\{\phi\})=\int d \vec{r}\left[V_{0}[\phi(\vec{r})]+\frac{K}{2}|\vec{\nabla} \phi(\vec{r})|^{2}\right],
$$

and we choose a monostable local potential $V_{0}(\phi)=\frac{a}{2} \phi^{2}$, with $a>0$. We also consider a nonconserved order parameter system in which the kinetic coefficient $\Gamma$ is only a function of the field and not of its spatial derivatives. According to the discussion above, we adopt the functional form,

$$
\Gamma(\phi)=1 /\left(1+c \phi^{2}\right),
$$

which means that fluctuations are larger in dilute regions and smaller in dense ones. In this particular case, and according to Eqs. (4) and (5), the local effective potential can be seen to become bistable for $\varepsilon_{0}>a / c$. One can then expect that, for a strong enough coupling $K$, a true phase transition towards an ordered state controlled by the noise intensity $\varepsilon$ exists. In order to check this expectation, we perform a Weiss mean-field analysis of the corresponding local effective potential [18]. The analysis is more easily done by considering a spatial discretization in a regular $d$-dimensional lattice with spacing $\Delta x=1$, i.e., $\phi\left(\vec{r}_{i}\right) \rightarrow \phi_{i}$, with $i$ the cell index. The gradient term is then approximated by the sum over nearest neighbors on the lattice in the standard way, $|\vec{\nabla} \phi|^{2} \rightarrow \sum_{j=1}^{d}\left(\phi_{j}-\phi_{i}\right)^{2}$. The mean-field approximation is tantamount to replacing the exact value of the neighbors by a mean-field common value $M$. In this way, the local effective potential becomes

$$
V_{\text {eff }}(\phi, M)=V_{0}(\phi)+\frac{\varepsilon_{0}}{2} \ln \Gamma(\phi)+d K(\phi-M)^{2},
$$

and depends on the yet unknown mean-field value $M$. This can be computed by using the consistency relation:

$$
M=\frac{\int_{-\infty}^{\infty} \phi e^{-V_{\text {eff }}(\phi, M) / \varepsilon} d \phi}{\int_{-\infty}^{\infty} e^{-V_{\text {eff }}(\phi, M) / \varepsilon} d \phi}
$$

which, in general, has to be solved numerically. The solution of this equation is shown as a solid line in Fig. 1 for the two-dimensional case. The result predicts a phase transition from disorder $(M=0)$ to order $(M \neq 0)$ as the noise intensity increases. This theoretical result has been confirmed by numerical simulations of a discretized version of model (2) in a two-dimensional square lattice. Following standard procedures of statistical mechanics, we have characterized the phase transition by means of two quantities: the intensive order parameter $M=\langle m\rangle$, where $m=\left|\sum_{i} \phi_{i}\right| / L^{2}$ is the absolute value of the spatial average of the field, and the generalized susceptibility

$$
\chi=L^{2} \frac{\left\langle m^{2}\right\rangle-\langle m\rangle^{2}}{\varepsilon},
$$

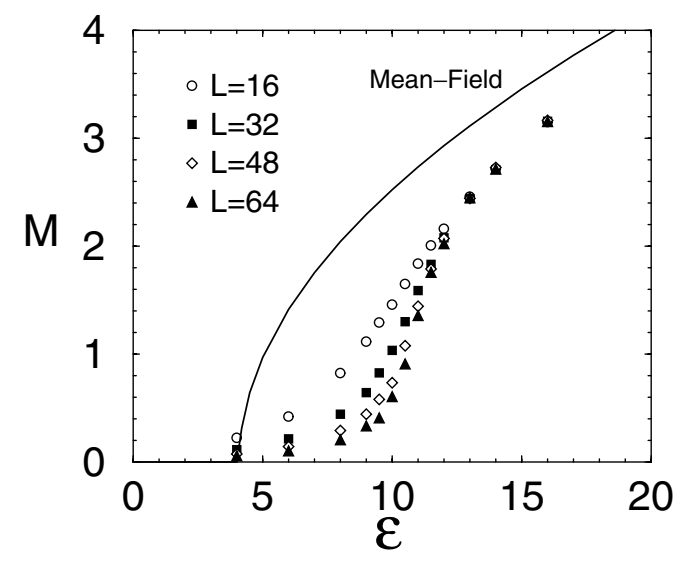

FIG. 1. Inverted phase diagram: intensive order parameter $M$ as a function of the noise intensity for $a=1, c=0.5, K=1$, and for four different system sizes. The continuous line is the Weiss mean-field result. 


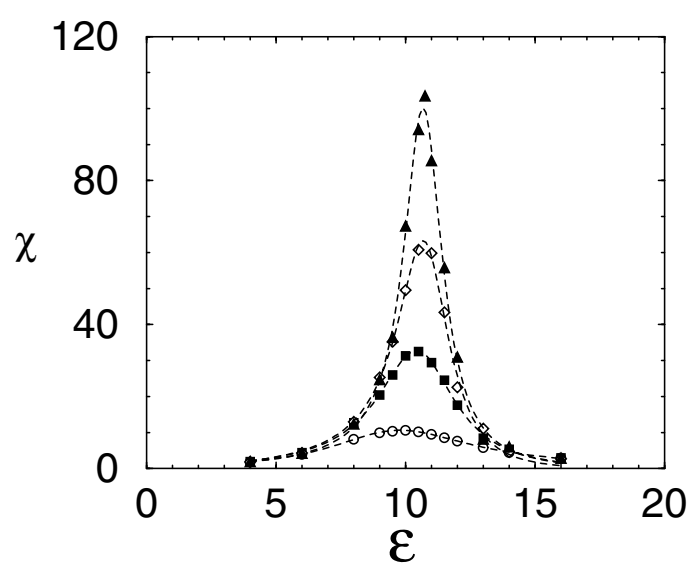

FIG. 2. Generalized susceptibility $\chi$ versus noise intensity for the case of Fig. 1.

which measures the relative fluctuations of the field in its steady state ( $\varepsilon$ plays the role of $K_{B} T$ in equilibrium phase transitions and $L$ is the linear system size). The numerical results are plotted in Figs. 1 and 2, which show the behavior of the order parameter $M$ and the susceptibility $\chi$, respectively, as the noise intensity $\varepsilon$ increases, for four different system sizes. The figures clearly denote the existence of a noise-induced ordering phase transition with the usual features of a second-order phase transition, in agreement with the theoretical analysis. Monte Carlo simulations of the system making use of the nonequilibrium effective free energy give completely compatible results. The position of the critical point has been computed both by extrapolating to infinite size the location of the maxima of the susceptibility and by means of a finite-size scaling analysis (see below). The two approaches lead to similar results.

As mentioned above, noise-induced phase transitions have been related thus far to an instability of the homogeneous phase at short times. This had been clearly manifested by a linear stability analysis of the equation for the first moment, and was understood as a renormalization of the linear forces due to the noise term. Let us now perform in our model a similar linear stability analysis of the homogeneous disordered state $\phi=0[19,20]$, and we will be able to show that this is not the mechanism that induces the phase transition in our model. The dynamical equation for the first statistical moment can be obtained from Eq. (2). In the linear approximation, it reads

$$
\frac{\partial\langle\phi\rangle}{\partial t}=-\left(a+\varepsilon_{0} c\right)\langle\phi\rangle+K \nabla^{2}\langle\phi\rangle,
$$

where Novikov's theorem has been applied to the term $\left\langle\Gamma(\phi)^{1 / 2} \xi\right\rangle$.

The contribution of the noise is in the same direction as the one from the local potential, and thus the homogeneous state $\phi=0$ is linearly stable. Hence, the physical mechanism responsible for the appearance of a new phase must be different from the one of previously known cases of noise-induced phase transitions. A simple interpretation can be found in terms of a balance between the role of the deterministic monostable local potential, which tends to take the system towards the disordered $(\phi=0)$ phase, and the stochastic motion, which is due to the fact that fluctuations are more intense in the disordered phase, and thus push the system away from it. The presence of a spatial coupling $K$ helps to break the symmetry of the homogeneous state.

A natural question arises concerning the universality class of the noise-induced phase transition described above. The availability of an exact nonequilibrium potential for this model, given by Eq. (5), allows us in a very simple way to analyze its universal behavior at the critical point. If an expansion of $\ln \Gamma(\phi)$, as chosen in Eq. (7), is performed on (5), the nonequilibrium effective potential can be seen to have a Ginzburg-Landau form with infinite higher-order (irrelevant) terms. Since the universality class of the Ginzburg-Landau model is that of the Ising model, we predict that this transition will belong to the $d$-dimensional Ising universality class. This is indeed confirmed by a finite-size scaling analysis of the numerical results. We make use of the following scaling laws, generalized from equilibrium statistical mechanics [1]:

$$
\begin{gathered}
M=L^{-\beta / \nu} \tilde{M}\left(\tilde{\varepsilon} L^{1 / \nu}\right), \\
\chi=L^{\gamma / \nu} \tilde{\chi}\left(\tilde{\varepsilon} L^{1 / \nu}\right),
\end{gathered}
$$

where $\tilde{M}$ and $\tilde{\chi}$ are the corresponding scaling functions, and $\tilde{\varepsilon}$ is the reduced control parameter, $\tilde{\varepsilon}=\left(1-\varepsilon / \varepsilon_{c}\right)$. The values of the critical exponents $\beta, \gamma$, and $\nu$ define the universality class. We have chosen the corresponding values of the 2D-Ising universality class, and plotted expressions (12) and (13) in Figs. 3 and 4. All data are seen to scale fairly well into the same curve in the region close to the critical point. As expected, those data corresponding to the smallest system size $(L=16)$ show the major discrepancies. Hence, we can conclude that this new kind

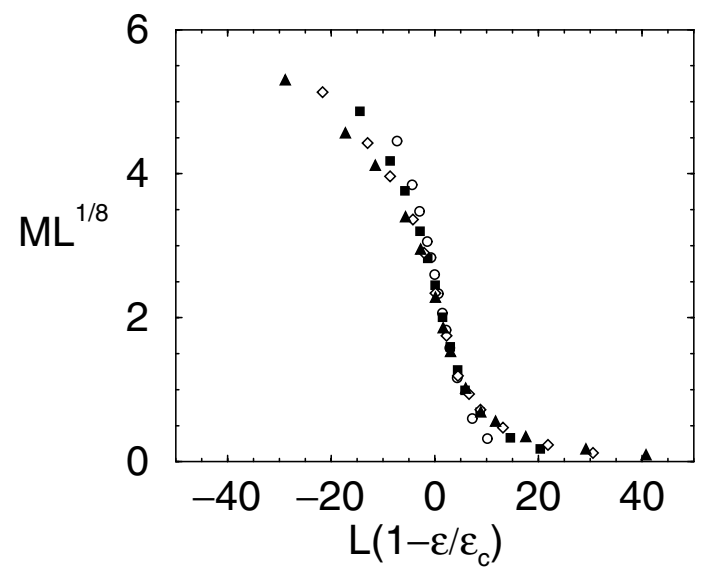

FIG. 3. Finite-size scaling function of the order parameter $M$ with the 2D-Ising critical exponents. 


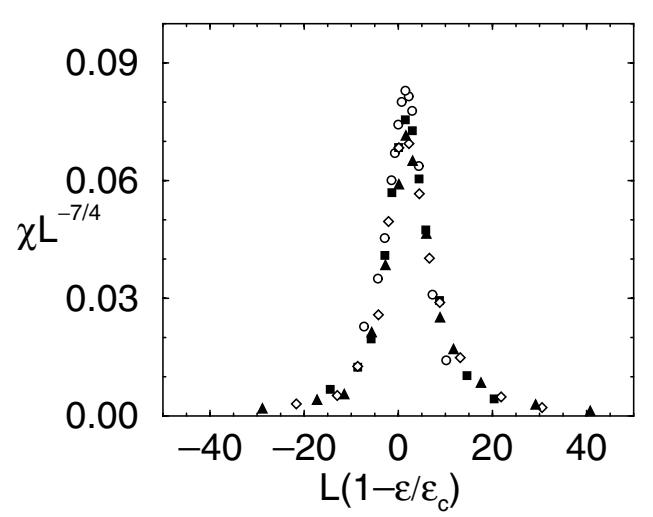

FIG. 4. Finite-size scaling function of the susceptibility $\chi$ with the 2D-Ising critical exponents.

of noise-induced phase transition belongs to the 2D-Ising universality class.

In conclusion, we have introduced a dynamical system that exhibits a nonequilibrium phase transition from disorder to order as the intensity of the noise is increased. As a consequence, the system is disordered below a certain amount of noise and can be considered a possible scenario, within the context of stochastic differential equations, for the appearance of a lower critical temperature in physical systems with an inverted phase diagram. At variance with other noise-induced nonequilibrium phase transitions reported previously, the physical mechanism inducing the transition is not a noise-induced short-time instability of the disordered state. Rather, we are able to show that the effective local potential, which can be obtained analytically, exhibits bistability as the intensity of the noise increases, whereas the deterministic one is monostable. Hence, the mechanism is seen to be given by a balance between deterministic forces and stochastic motion. Our model shows that it is possible to obtain a noise-induced phase transition by coupling adequately zero-dimensional systems undergoing a noise-induced transition. We have argued that this type of coupling is consistent with a mesoscopic description in terms of fields, kinetic coefficients, and the fluctuation-dissipation relation. Our findings have been sustained by numerical simulations in a twodimensional lattice model. Finally, by studying the exact stationary probability distribution, we are able to show that the model belongs to the same universality class as the Ising model.

We acknowledge the Dirección General de Enseñanza Superior (Spain) for financial support under Projects No. PB97-0141-C02-01, No. PB98-0935, No. BFM2000-0624, and No. BFM2000-1108, and the Fundació Catalana per a la Recerca for computing resources.
[1] J. García-Ojalvo and J. M. Sancho, Noise in Spatially Extended Systems (Springer-Verlag, New York, 1999).

[2] J. García-Ojalvo, A. Hernández-Machado, and J. M. Sancho, Phys. Rev. Lett. 71, 1542 (1993); J. M. R. Parrondo, C. Van den Broeck, J. Buceta, and J. de la Rubia, Physica (Amsterdam) 224A, 153 (1996).

[3] P. Jung and G. Mayer-Kress, Phys. Rev. Lett. 74, 2130 (1995); S. Kádár, J. Wang, and K. Showalter, Nature (London) 391, 770 (1998); H. Hempel, L. Schimansky-Geier, and J. García-Ojalvo, Phys. Rev. Lett. 82, 3713 (1999).

[4] J. García-Ojalvo, A. M. Lacasta, F. Sagués, and J. M. Sancho, Europhys. Lett. 50, 427 (2000).

[5] J. García-Ojalvo and L. Schimansky-Geier, Europhys. Lett. 47, 298 (1999); M. G. Zimmermann, R. Toral, O. Piro, and M. San Miguel, Phys. Rev. Lett. 85, 3612 (2000).

[6] A. A. Zaikin, J. Kurths, and L. Schimansky-Geier, Phys. Rev. Lett. 85, 227 (2000).

[7] C. Van den Broeck, J. M. R. Parrondo, and R. Toral, Phys. Rev. Lett. 73, 3395 (1994); C. Van den Broeck, J. M. R. Parrondo, R. Toral, and R. Kawai, Phys. Rev. E 55, 4084 (1997).

[8] J. M. Sancho and J. García-Ojalvo, in Stochastic Processes in Physics, Chemistry, and Biology, edited by J. A. Freund and T. Pöschel, Lecture Notes in Physics Vol. 557 (Springer-Verlag, Berlin, 2000).

[9] W. Horsthemke and R. Lefever, Noise-Induced Transitions (Springer-Verlag, Berlin, 1984).

[10] M. San Miguel and R. Toral, in Instabilities and Nonequilibrium Structures VI, edited by E. Tirapegui, J. Martínez, and R. Tiemann (Kluwer, Dordrecht, 2000).

[11] H. L. Snyder, P. Meakin, and S. Reich, Macromolecules 16, 757 (1983).

[12] R. Montagne, E. Hernández-García, and M. San Miguel, Physica (Amsterdam) 96D, 47 (1996).

[13] J. S. Langer, Ann. Phys. (N.Y.) 65, 53 (1971).

[14] K. Kitahara and M. Imada, Suppl. Prog. Theor. Phys. 64, 65 (1978).

[15] G. Martin, Phys. Rev. B 41, 2279 (1990).

[16] K. Kitahara, Y. Oono, and D. Jasnow, Mod. Phys. Lett. B 2, 765 (1988).

[17] As it is well known, calculations in a continuum space in the presence of a multiplicative white noise have to be considered with care $[1,10]$. Therefore, we interpret our noise here to have a cutoff at short length scales, according to standard approaches in nonequilibrium critical dynamics. Using, for instance, a regular lattice of spacing $\Delta x$ for regularization, we find $\varepsilon=\varepsilon_{0} / \Delta x^{d}$. More details on our calculations will be published elsewhere.

[18] C. Van den Broeck, J. M. R. Parrondo, J. Armero, and A. Hernández-Machado, Phys. Rev. E 49, 2639 (1994).

[19] A. Becker and L. Kramer, Phys. Rev. Lett. 73, 955 (1994); Physica (Amsterdam) 90D, 408 (1995).

[20] M. Ibañes, J. García-Ojalvo, R. Toral, and J. M. Sancho, in Stochastic Processes in Physics, Chemistry, and Biology (Ref. [8]). 